\title{
Investigating the impact of on-chip interconnection noise on dynamic thermal management efficiency
}

\begin{abstract}
Dynamic Thermal Management (DTM) emerged as a solution to address the reliability challenges with thermal hotspots and unbalanced temperatures. DTM efficiency is highly affected by the accuracy of the temperature information presented to the DTM manager. This work aims to investigate the effect of inaccuracy caused by the deep sub-micron (DSM) noise during the transmission of temperature information to the manager on DTM efficiency. A simulation framework has been developed and results show up to 62\% DTM performance degradation under DSM noise. The finding highlights the importance of further research in providing reliable on-chip data transmission in DTM application.
\end{abstract}

Keyword: DTM; DSM noise; Performance 